\title{
A Note on Tsuji's Criterion for Numerical Triviality
}

\author{
Shigetaka Fukuda \\ Faculty of Education, Gifu Shotoku Gakuen University, Gifu, Japan \\ Correspondence should be addressed to Shigetaka Fukuda; fukuda@gifu.shotoku.ac.jp \\ Received 16 October 2021; Accepted 14 December 2021; Published 19 January 2022 \\ Academic Editor: Li Guo \\ Copyright (C) 2022 Shigetaka Fukuda. This is an open access article distributed under the Creative Commons Attribution License, \\ which permits unrestricted use, distribution, and reproduction in any medium, provided the original work is properly cited. \\ In this study, we give an alternative and elementary proof to Tsuji's criterion for a Cartier divisor to be numerically trivial.
}

\section{Introduction}

In this article, every algebraic variety is proper over the field of complex numbers $\mathbf{C}$.

In 1970s, Iitaka [1] initiated the classification theory of higher dimensional algebraic varieties by using the pluricanonical systems. In 1980s, Mori [2] deepened the Iitaka theory by cutting off the subvarieties of elliptic type.

In [3], Tsuji gave an interesting and useful criterion for a Cartier divisor to be numerically trivial.

Theorem 1 (Tsuji [[3], Lemma 5.1], cf. Bauer et al. [[4], Theorem 2.4]). Let $f: M \longrightarrow B$ a surjective morphism between complete varieties. Let $L$ be a nef Cartier divisor on $M$ and $W$ some subvarieties of $M$, such that $f(W)=B$ and $B_{0} a$ subset of $B$ which is a union of countably many proper Zariski-closed subsets. Assume that

(1) for some $b \in B,(L, C)=0$ for every curve $C$ on $f^{-1}(b)$

(2) $(L, C)=0$ for every irreducible curve $C$ on $W$, such that $f(C) \nsubseteq B_{0}$

Then, $L$ is numerically trivial.

Tsuji's criterion for numerical triviality is one of the basic tools to decompose every algebraic variety into the varieties of elliptic type, of parabolic type, and of hyperbolic type by cutting off the varieties of parabolic type [5-7].

Tsuji's proof [3] is analytic and the proof [4] by Bauer et al. is algebraic.

In this research note, we give an alternative and elementary proof to Tsuji's criterion (Theorem 1). The argument (Subcase 7), which uses the following corollary of the Hodge index theorem, due to Bauer et al. is essential.

Lemma 1 (cf. [[4], Proposition 2.5]). Let $f: M \longrightarrow B$ be a surjective morphism from a complete surface $M$ to a complete curve B. Let L be a nef Cartier divisor on M. Assume that

(1) For some $b \in B,(L, C)=0$ for every curve $C$ on $f^{-1}(b)$

(2) $(L, W)=0$ for some irreducible curve $W$ on $M$, such that $f(W)=B$

Then, $L$ is numerically trivial.

Remark 1. In the statement of Lemma 1, condition (1) immediately implies that $L$ is numerically trivial on every general fiber of the morphism $f$ by considering the flattening. By the normalization, the Stein factorization, and the desingularization, the article ([4], Proposition 2.5), for an algebraically fibered surface, implies the assertion of Lemma 1.

\section{Elementary Proof of Main Theorem 1}

Proof. We prove the assertion by induction on $(\operatorname{dim} M, \operatorname{dim} B)$.

First, we take a commutative diagram as shown in Figure 1 with the following properties:

(1) $M^{\prime}$ and $B^{\prime}$ are nonsingular projective varieties

(2) $\mu$ is a birational morphism

(3) $v$ is a generically finite morphism 


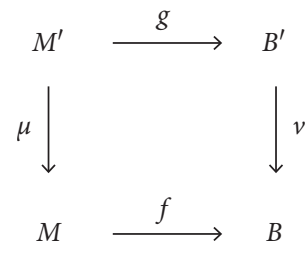

Figure 1: The Stein factorization for $f$ is as follows.

(4) $g$ is a morphism with only connected fibers

There exists some irreducible component $W^{\prime}$ of $\mu^{-1}(W)$, such that $g\left(W^{\prime}\right)=B^{\prime}$. We set $L^{\prime}:=\mu^{*} L$.

The locus $g\left(\cup\left\{C^{\prime} \mid C^{\prime}\right.\right.$ is an irreducible curve on $M^{\prime}, g$ $\left(C^{\prime}\right)$ is a point, and the intersection number $\left.\left.\left(L^{\prime}, C^{\prime}\right)>0\right\}\right)$ is included in a union of at most countably many proper Zariski-closed subsets of $B^{\prime}$ (Proposition 1). Thus, we obtain a union $B_{0}^{\prime} \supseteq v^{-1}\left(B_{0}\right)$ of countably many proper Zariskiclosed subsets of $B^{\prime}$ with the following two properties:

(1) $L^{\prime}$ is numerically trivial on every fiber of $g$ over $B^{\prime} \backslash B_{0}^{\prime}$

(2) $\left(L^{\prime}, C_{W}^{\prime}\right)=0$ for every irreducible curve $C_{W}{ }^{\prime}$ on $W^{\prime}$, such that $g\left(C_{W}^{\prime}\right) \nsubseteq B_{0}^{\prime}$

It suffices to prove that $\left(L^{\prime}, C^{\prime}\right)=0$ for every irreducible curve $C^{\prime}$ on $M^{\prime}$. We fix an irreducible curve $C^{\prime}$ on $M^{\prime}$.

Case 1. $g\left(C^{\prime}\right) \nsubseteq B_{0}^{\prime}$. This case divides into Subcases 1 and 2 .

Subcase 1. $g\left(C^{\prime}\right) \nsubseteq B_{0}^{\prime}$ and $g\left(C^{\prime}\right)$ is a point.

We have $\left(L^{\prime}, C^{\prime}\right)=0$ from $(1)$.

Subcase 2. $\mathrm{g}\left(\mathrm{C}^{\prime}\right) \nsubseteq \mathrm{B}_{0}^{\prime}$ and $\mathrm{g}\left(\mathrm{C}^{\prime}\right)$ is a curve. This subcase divides into Subcases 3 and 7.

Subcase 3. $g\left(C^{\prime}\right) \nsubseteq B_{0}^{\prime}, g\left(C^{\prime}\right)$ is a curve and $\operatorname{dim} B=1$. This subcase divides into Subcases 4,5 , and 6 .

We note that $g\left(W_{1}\right)=g\left(C^{\prime}\right)=B^{\prime}$ for some irreducible curve $W_{1}$ on $W^{\prime}$.

Subcase 4. $g\left(C^{\prime}\right) \nsubseteq B_{0}^{\prime}, g\left(C^{\prime}\right)$ is a curve, $\operatorname{dim} B=1$, and $\operatorname{dim} M=1$.

$$
M^{\prime}=W^{\prime}=W_{1}=C^{\prime} \text {. Thus, }\left(L^{\prime}, C^{\prime}\right)=0 .
$$

Subcase 5. $g\left(C^{\prime}\right) \nsubseteq B_{0}^{\prime}, g\left(C^{\prime}\right)$ is a curve, $\operatorname{dim} B=1$, and $\operatorname{dim} M=2$.

Because $\left(L^{\prime}, W_{1}\right)=0$ from (2), Lemma 1 implies that $L^{\prime}$ is numerically trivial, and thus, $\left(L^{\prime}, C^{\prime}\right)=0$.

Subcase 6. $g\left(C^{\prime}\right) \nsubseteq B_{0}^{\prime}, g\left(C^{\prime}\right)$ is a curve, $\operatorname{dim} B=1$, and $\operatorname{dim} M \geqq 3$.

Because the codimension $\operatorname{codim}\left(W_{1} \cup C^{\prime}, M^{\prime}\right) \geqq 2$, we have an irreducible hyperplane section $H$ of $M^{\prime}$ that includes $W_{1}$ and $C^{\prime}$ (Proposition 2). Then, $\left.L^{\prime}\right|_{H}$ is numerically trivial from the induction hypothesis. Consequently, $\left(L^{\prime}, C^{\prime}\right)=0$.

Subcase 7 (cf. [[4], 2.1.2]). $g\left(C^{\prime}\right) \nsubseteq B_{0}^{\prime}, g\left(C^{\prime}\right)$ is a curve, and $\operatorname{dim} B \geqq 2$.
Let $S:=\left\{S_{i}\right\}$ be the set of irreducible components of $g^{-1}\left(g\left(C^{\prime}\right)\right)$. We note that $g\left(W_{1}\right)=g\left(C^{\prime}\right)$ for some irreducible curve $W_{1}$ on $W^{\prime}$. Thus, $\left.L^{\prime}\right|_{S_{1}}$ is numerically trivial for some $S_{1} \in S$, such that $S_{1} \supseteq W_{1}$ from the property (2) and from the induction hypothesis.

If $g\left(\cup_{m \neq 1} S_{m}\right)=g\left(C^{\prime}\right)$, then $g\left(S_{1} \cap\left(\cup_{m \neq 1} S_{m}\right)\right)=g\left(C^{\prime}\right)$ from the connectedness of fibers of $g$, and therefore, $g\left(S_{1} \cap S_{2}\right)=g\left(C^{\prime}\right)$ for some $S_{2} \in S$.

Thus, $\operatorname{dimg}\left(\cup_{m \neq 1} S_{m}\right) \leqq 0$ or $g\left(S_{1} \cap S_{2}\right)=g\left(C^{\prime}\right)$.

If $g\left(S_{1} \cap S_{2}\right)=g\left(C^{\prime}\right)$ and $g\left(\cup_{m \neq 1,2} S_{m}\right)=g\left(C^{\prime}\right)$, then $g\left(\left(S_{1} \cup S_{2}\right) \cap\left(\cup_{m \neq 1,2} S_{m}\right)\right)=g\left(C^{\prime}\right)$ from the connectedness of fibers of $g$, and therefore, $g\left(\left(S_{1} \cup S_{2}\right) \cap S_{3}\right)=g\left(C^{\prime}\right)$ for some $S_{3} \in S$. From this argument, we obtain the following properties:

(1) $g\left(S_{1}\right)=g\left(S_{2}\right)=\cdots=g\left(S_{k}\right)=g\left(C^{\prime}\right)$

(2) $g\left(\left(S_{1} \cup S_{2} \cup \cdots \cup S_{i-1}\right) \cap S_{i}\right)=g\left(C^{\prime}\right)$ for all $i$ with $1 \leqq i \leqq k$

(3) $\operatorname{dimg}\left(\cup_{i \neq 1,2, \ldots k} S_{i}\right) \leqq 0$

The fact that $\left.L^{\prime}\right|_{S}$ is numerically trivial and that $g\left(S_{1} \cap S_{2}\right)=g\left(C^{\prime}\right)$ implies that $\left.L^{\prime}\right|_{S_{2}}$ is numerically trivial from the induction hypothesis. The fact that $\left.L^{\prime}\right|_{S_{1} \cup S_{2}}$ is numerically trivial and that $g\left(\left(S_{1} \cup S_{2}\right) \cap S_{3}\right)=g\left(C^{\prime}\right)$ implies that $\left.L^{\prime}\right|_{S_{3}}$ is numerically trivial from the induction hypothesis. This argument implies that $\left.L^{\prime}\right|_{S_{1} \cup S_{2} \cup \ldots \cup S_{k}}$ is numerically trivial. Because dimg $\left(\cup_{i \neq 1,2, \ldots k} S_{i}\right) \leqq 0$, we have that $C^{\prime} \subseteq S_{1} \cup S_{2} \cup \cdots \cup S_{k}$. Consequently, $\left(L^{\prime}, C^{\prime}\right)=0$.

Case 2. $g\left(C^{\prime}\right) \subseteq B_{0}^{\prime}$. This case divides into Subcases 8 and 11 .

Subcase 8. $g\left(C^{\prime}\right) \subseteq B_{0}^{\prime}$ and $\operatorname{dim} M=2$. This subcase divides into Subcases 9 and 10 .

Subcase 9. $g\left(C^{\prime}\right) \subseteq B_{0}^{\prime}$, $\operatorname{dim} M=2$, and $\operatorname{dim} B=1$.

Lemma 1 implies that $L^{\prime}$ is numerically trivial, and thus, $\left(L^{\prime}, C^{\prime}\right)=0$.

Subcase 10. $g\left(C^{\prime}\right) \subseteq B_{0}^{\prime}$, $\operatorname{dim} M=2$, and $\operatorname{dim} B=2$.

Because $W^{\prime}=M^{\prime}$, we have that $\left(L^{\prime}, H\right)=0$ for an irreducible hyperplane section $H$ of $M^{\prime}$ from the property (2) of the divisor $L^{\prime}$. The Hodge index theorem implies that $L^{\prime}$ is numerically trivial. Thus, $\left(L^{\prime}, C^{\prime}\right)=0$.

Subcase 11. $g\left(C^{\prime}\right) \subseteq B_{0}^{\prime}$ and $\operatorname{dim} M \geqq 3$.

Because the codimension codim $\left(C^{\prime}, M^{\prime}\right) \geqq 2$, there exists an irreducible hyperplane section $H$ of $M^{\prime}$ that includes $C^{\prime}$ (Proposition 2). We may assume that $g(H) \nsubseteq B_{0}^{\prime}$. Note that, from Case $1,\left(L, C^{\prime \prime}\right)=0$ for every irreducible curve $C^{\prime \prime}$ on $H$, such that $g\left(C^{\prime \prime}\right) \nsubseteq B_{0}^{\prime}$. Thus, $\left.L^{\prime}\right|_{H}$ is numerically trivial from the induction hypothesis. Consequently, $\left(L^{\prime}, C^{\prime}\right)=0 . \square$

\section{Appendix}

In this appendix, we state two elementary propositions and their proofs, which are well known to the experts, for the readers' convenience. 
Proposition 1. Let $f: M \longrightarrow B$ be a surjective morphism between projective varieties and $L$ a nef Cartier divisor on $M$.

We assume that for some $b \in B$, the intersection number $(L, C)=0$ for every irreducible curve $C$ on $f^{-1}(b)$.

Then, the locus $f(\cup\{C \mid C$ is an irreducible curve on $M$, $f(C)$ is a point, and the intersection number $(L, C)>0\})$ is included in a union of at most countably many proper Zariski-closed subsets of $B$.

Proof. There exists some ample divisor $A$ on $B$. Assume that $C$ is an irreducible curve on $M$, such that $\left(f^{*} A, C\right)=$ 0 (i.e., $f(C)$ is a point) and that $(L, C)>0$. There exists some irreducible component $W$ of the universal scheme for the Hilbert scheme Hilb $(M)$ of $M$, such that $W$ includes $p_{2}^{-1}([C])$, where $[C]$ is the point $(\in \operatorname{Hilb}(M))$, which represents the subscheme $C$ of $M$, and Figure 2 shows the projections $p_{1}$ and $p_{2}$ and the property that $\operatorname{dim} W=\operatorname{dim}_{2}(W)+1$. We set $T:=p_{2}(W)$.

First, we consider the normalization $n_{1}: W_{n} \longrightarrow W$, $n_{2}: T_{n} \longrightarrow T$, and $n_{3}: W_{n} \longrightarrow T_{n}$ of the morphism $\left.p_{2}\right|_{W}: W \longrightarrow T$. Next, consider the Stein factorization $W_{n} \stackrel{s_{1}}{\longrightarrow} T^{\prime} \stackrel{s_{2}}{\longrightarrow} T_{n}$ of the morphism $n_{3}: W_{n} \longrightarrow T_{n}$.

Last, consider the flattening $f_{1}: W^{\prime \prime} \longrightarrow W_{n}$, $f_{2}: T^{\prime \prime} \longrightarrow T^{\prime}$, and $f_{3}: W^{\prime \prime} \longrightarrow T^{\prime \prime}$ of the morphism $s_{1}: W_{n} \longrightarrow T^{\prime}$, where the morphism $f_{2}$ is birational and the variety $T^{\prime \prime}$ is nonsingular. We note that the morphism $f_{3}: W^{\prime \prime} \longrightarrow T^{\prime \prime}$ is flat and with only connected fibers.

We put $h:=n_{1} f_{1}$.

Thus, we have the commutative diagram, as shown in Figure 3.

From the flatness of the morphism $f_{3}: W^{\prime \prime} \longrightarrow T^{\prime \prime}$, the intersection number $\left(h^{*} p_{1}^{*} f^{*} A, F^{\prime \prime}\right)>0$ for every fiber $F^{\prime \prime}$ of the morphism $f_{3}: W^{\prime \prime} \longrightarrow T^{\prime \prime}$ because $\left(p_{1}^{*} f^{*} A, p_{2}^{-1}([C])\right)=0$. Thus, for every fiber $F^{\prime \prime}$ of the morphism $f_{3}: W^{\prime \prime} \longrightarrow T^{\prime \prime}$, the morphism $f$ contracts $p_{1} h\left(F^{\prime \prime}\right)$ to one point from the connectedness of $F^{\prime \prime}$. In other words, $p_{1} h\left(F^{\prime \prime}\right)$ is included in some fiber of $f$.

There exists some ample divisor $A^{\prime}$ on $W$. Of course, $\left(A^{\prime}, F\right)>0$ for every curve $F$ on $W$. Because the morphism $h$ is birational, we have that $h\left(F^{\prime \prime \prime}\right)$ is not a point (i.e., $\left(h^{*} A^{\prime}, F^{\prime \prime \prime}\right)>0$ ) for some fiber $F^{\prime \prime \prime}$ of $f_{3}: W^{\prime \prime} \longrightarrow T^{\prime \prime}$. From the flatness, $\left(h^{*} A^{\prime}, F^{\prime \prime}\right)>0$ for every fiber $F^{\prime \prime}$ of $f_{3}: W^{\prime \prime} \longrightarrow T^{\prime \prime}$. Thus, every fiber $F^{\prime \prime}$ of $f_{3}: W^{\prime \prime} \longrightarrow T^{\prime \prime}$ cannot be contracted to a point by the morphism $h$.

There exists some fiber $F_{1}^{\prime \prime}$ of $f_{3}: W^{\prime \prime} \longrightarrow T^{\prime \prime}$, such that $h^{-1}\left(p_{2}^{-1}([C])\right) \cap F_{1}^{\prime \prime} \neq \varnothing$. Then, $F_{1}^{\prime \prime} \subset h^{-1}\left(p_{2}^{-1}([C])\right.$ because the morphism $p_{2} h$ maps $F_{1}^{\prime \prime}$ to a point $[C] \in T$. Consequently, $h\left(F_{1}^{\prime \prime}\right)=p_{2}^{-1}([C])$ because $F_{1}^{\prime \prime}$ does not contract to a point by the morphism $h$. Thus, $\left(h^{*} p_{1}^{*} L, F_{1}^{\prime \prime}\right)>0$. From the flatness of the morphism $f_{3}: W^{\prime \prime} \longrightarrow T^{\prime \prime}$, the intersection number is $\left(h^{*} p_{1}^{*} L, F^{\prime \prime}\right)>0$ for every fiber $F^{\prime \prime}$ of the morphism $f_{3}: W^{\prime \prime} \longrightarrow T^{\prime \prime}$.

We note that every fiber of $f_{3}: W^{\prime \prime} \longrightarrow T^{\prime \prime}$ is mapped in some fiber of $\left.p_{2}\right|_{W}: W \longrightarrow T$. In other words, every fiber of $\left.p_{2}\right|_{W}$ is swept out by fibers of $f_{3}$.

So, for every fiber $F$ of $\left.p_{2}\right|_{W}: W \longrightarrow T$, the locus $p_{1}(F)$ is swept out by connected curves $C^{\prime}$, such that $f\left(C^{\prime}\right)$ is one

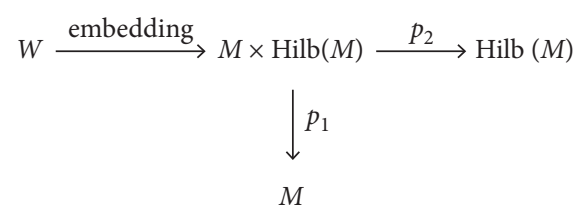

Figure 2: The deformation of $C$ in $M$.

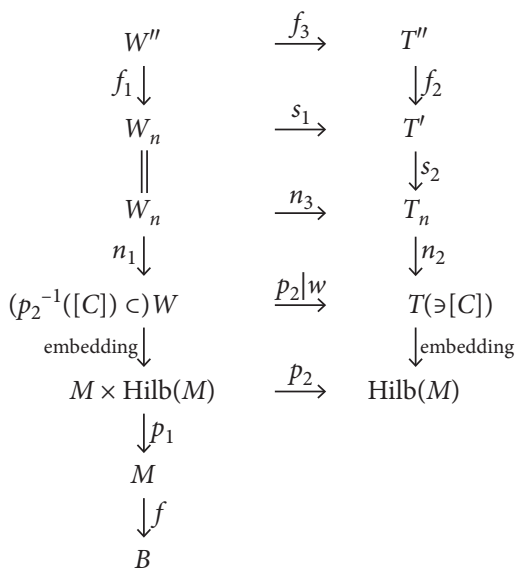

Figure 3: The flattening of the deformation.

point and that the intersection number $\left(L, C^{\prime}\right)>0$. We note that we consider $p_{1} h\left(F^{\prime \prime}\right)$ as $C^{\prime}$ and that $C^{\prime}=p_{1} h\left(F^{\prime \prime}\right)=$ $\operatorname{Supp}\left(\left(p_{1} h\right)_{*} F^{\prime \prime}\right)$ from the connectedness of $F^{\prime \prime}$. Thus, $b \notin f\left(p_{1}(F)\right)$. Consequently, $b \notin f\left(p_{1}(W)\right)$. In other words, $p_{1}(W)$ is disjoint with $f^{-1}(b)$.

The countability of the irreducible components of the Hilbert scheme Hilb(M) of $M$ implies the assertion.

Proposition 2. Let $M$ be a nonsingular projective variety and $C$ a Zariski-closed subset with codimension $\operatorname{codim}(C, M) \geq 2$. Then, there exists some irreducible hyperplane section $H$, such that $H^{C}$.

Proof. We take some ample divisor $A$ on $M$. We have a birational morphism $f: M^{\prime} \longrightarrow M$, such that $M^{\prime}$ is a nonsingular projective variety, that $f^{-1}(C)$ is divisorial with only simple normal crossings and that there exists an effective divisor $C_{0}$ with the property that $\operatorname{Supp}\left(C_{0}\right)=f^{-1}(C)$ and $-C_{0}$ is $f$-ample. Then, $m f^{*} A-C_{0}$ is ample for a sufficiently large integer $m$. For a sufficiently large and divisible integer $l$, the divisor $\operatorname{lm} A$ is very ample, and there exists a member $H_{0} \in\left|l\left(m f^{*} A-C_{0}\right)\right|$ which is very ample and irreducible. We put $H:=f_{*}\left(H_{0}+l C_{0}\right)$. Then, $H \in|\operatorname{lm} A|$.

The locus $f^{-1}(H)$ coincides with $\operatorname{Supp}\left(f^{*} H\right)=$ $\operatorname{Supp}\left(H_{0}+l C_{0}\right)$. Thus, $f^{-1}(H)^{f^{-1}(C)}$.

\section{Data Availability}

No data were used to support this study.

\section{Disclosure}

The updated version of the manuscript is presented in arXiv: $2109.02034 v 1([8])$. 


\section{Conflicts of Interest}

The author declares that there are no conflicts of interest.

\section{Acknowledgments}

The author was supported by the research grant of Gifu Shotoku Gakuen University in the years 2019 and 2020.

\section{References}

[1] S. Iitaka, "On D-dimensions of algebraic varieties," Journal of the Mathematical Society of Japan, vol. 23, pp. 356-373, 1971.

[2] S. Mori, "Threefolds whose canonical bundles are not numerically effective," Annals of Mathematics, vol. 116, no. 1, pp. 133-176, 1982.

[3] H. Tsuji, "Numerically trivial fibrations," 2000, https://arxiv. org/abs/math/0001023.

[4] T. Bauer, F. Campana, T. Eckl et al., "A reduction map for Nef line bundles," in Complex Geometry, pp. 27-36, Springer, Berlin, Germany, 2002.

[5] F. Ambro, "Nef dimension of minimal models," Mathematische Annalen, vol. 330, pp. 309-322, 2004.

[6] F. Ambro, "The moduli b-divisor of an lc-trivial fibration," Compositio Mathematica, vol. 141, no. 2, pp. 385-403, 2005.

[7] S. Fukuda, "Tsuji's numerically trivial fibrations and abundance," Far East Journal of Mathematical Sciences, vol. 5, pp. 247-257, 2002.

[8] S. Fukuda, "A note on Tsuji's criterion for numerical triviality," 2021, https://arxiv.org/abs/2109.02034. 